\title{
Isolation, Characterization, and Emendation of Description of Angiococcus disciformis (Thaxter 1904) Jahn 1924 and Proposal of a Neotype Strain
}

\author{
LEONARD A. HOOK,' JOHN M. LARKIN, ${ }^{1}$ AND ELLIS R. BROCKMAN ${ }^{2}$ \\ Department of Microbiology, Louisiana State University, Baton Rouge, Louisiana $70803,{ }^{1}$ and Department \\ of Biology, Central Michigan University, Mt. Pleasant, Michigan $48859^{2}$
}

Strain CMU-1, identified as a member of Angiococcus disciformis (Thaxter) Jahn 1924, was isolated from aquatic habitats in the fossa region of Davis Lake (Vestaburg Bog), Michigan. The myxobacter produced orange fruiting bodies consisting of numerous disk-shaped to oblate spheroid-shaped sporangia (average, 40 by $27 \mu \mathrm{m} ; 15 \mu \mathrm{m}$ thick). The sporangia comprising the fruiting body occurred singly or were clumped in irregular masses upon a solid substrate. Mature myxospores ranged in shape from irregular spheres $(1 \mu \mathrm{m}$ in diameter) to ovals ( 1 by $1.5 \mu \mathrm{m}$ ). Vegetative rods were motile by gliding and measured 0.5 to 0.75 by 3 to $14 \mu \mathrm{m}$. The guanine plus cytosine content of the deoxyribonucleic acid extracted from $A$. disciformis CMU-1 was $68.4 \mathrm{~mol} \%$. Strain CMU-1 (ATCC 33172) has the properties recorded in the original description of Myxococcus disciformis by Thaxter, and it is herein proposed as the neotype strain of this organism. Because the myxospores were contained within sporangia, the organism cannot belong to the genus Myxococcus, and we propose that it properly belongs in the genus Angiococcus Jahn 1924.

Thaxter (13) first described Myxococcus disciformis in 1904, having detected the myxobacter on muskrat dung from Massachusetts and deer dung from New Hampshire. Presumably based on his observation of the irregularly spherical myxospores of the organism, the species was placed in the family Myxococcaceae, the members of which exhibit rounded myxospores (9). Jahn (4) created a new genus, Angiococcus, for this organism on the basis of the description by Thaxter of the distinct, thin, slightly wrinkled sporangium wall that enclosed the myxospores (13). In this new position, the name of the organism was Angiococcus disciformis (Thaxter) Jahn. Krzemieniewska and Krzemieniewski (6) reported the occurrence of $A$. disciformis on rabbit and goat dung and from Carpathian soil. They published a more thorough description of the species (6) and the first photomicrographs of the fruiting body and sporangia (7). They also reported the occurrence of $A$. disciformis in forest soil (pH 3.6 to 5.3) associated with Oxalis, Vaccinium, and Sphagnum and in bog soil $(\mathrm{pH}$ 3.8 to 4.8 ) dominated by Sphagnum and Carex (7).

Peterson and McDonald (12) were unable to isolate $A$. disciformis, and since no specimens of the genus existed, they recommended that the genus Angiococcus be dropped. Based on this recommendation and on the fact that detection of the species had not been reported since 1927,
McCurdy (9) omitted the genus Angiococcus from the 8th edition of Bergey's Manual and listed the organism as Myxococcus disciformis, species incertae sedis.

Recently, $A$. disciformis was detected abundantly in aquatic habitats of an alkaline $\mathrm{pH}$ bog (3). Here, we report the isolation of Angiococcus disciformis strain CMU-1 from the fossa region of Davis Lake (Vestaburg Bog), located in central lower Michigan. In addition, the organism has been characterized, and a culture of it has been deposited in the American Type Culture Collection under the number 33172.

\section{MATERIALS AND METHODS}

Sample collection. Sediment-laden water samples were collected from the fossa region of Davis Lake (Vestaburg Bog), an alkaline peat bog, as described previously (3). A particulate inoculum was obtained either by centrifugation of the water samples at 5,000 $\times g$ for 30 min or by filtering the water through several membrane filters (type HA, $0.45-\mu \mathrm{m}$ pore size; Millipore Corp., Bedford, Mass.) to collect the sediment on the membrane surface.

Isolation and maintenance of cultures. Three culture media were used for the isolation of $A$. disciformis: (i) mineral salts agar overlaid with a $7-\mathrm{cm}$ disk of sterile Whatman no. 1 filter paper (1); (ii) mineral salts agar streaked with a heavy suspension of autoclaved bakers' yeast (1); and (iii) dilute nutrient agar which contained, per liter of distilled water, $0.5 \mathrm{~g}$ of Nutrient Broth and $15 \mathrm{~g}$ of agar (Difco Laboratories, Detroit, Mich.). All media contained $0.025 \%$ cyclohex- 
imide (Acti-dione; Upjohn Co., Kalamazoo, Mich.) (1). The filter paper and bakers' yeast plates were inoculated with small amounts (approximately $25 \mathrm{mg}$ ) of sediment with a cotton swab (1). The filter paper disks provided a carbon source for cellulolytic bacteria which, in turn, served as a food source for the enrichment of several species of myxobacteria, including $A$. disciformis (3). The scraped membrane filters were placed grid side up on the surface of dilute nutrient agar plates. All plates were incubated at room temperature $\left(20\right.$ to $\left.22^{\circ} \mathrm{C}\right)$ and were examined periodically with a dissecting microscope for the presence of fruiting bodies. A pure culture of Angiococcus disciformis strain CMU-1 was obtained by repeated transfer of the leading edge of a vegetative swarm onto fresh media (11). Other attempts toward the isolation of $A$. disciformis which failed to separate the contaminants from the slime matrix involved homogenizing fruiting bodies picked from filter paper primary enrichment plates and streaking the cell suspension onto dilute nutrient agar. Purity of the isolate was tested by microscopic examination of the vegetative colony and by inoculating subcultures of the isolate into nutrient broth and checking for growth of contaminants after $48 \mathrm{~h}$ of incubation at $35^{\circ} \mathrm{C}$. A. disciformis strain CMU1 was maintained alternately on dilute nutrient agar and on a fourth medium consisting of mineral salts with a sterile rabbit dung pellet embedded in the agar (11).

Biochemical tests. Physiological characteristics of A. disciformis strain CMU-1 were determined by standard biochemical procedures (2). To test for catalase and cytochrome oxidase production, and for agar etching, vegetative colonies were grown on dilute nutrient agar. Congo red dye absorption by colonies grown on dilute nutrient agar was done by the method of McCurdy (8). The hydrolysis of casein and starch was determined by adding each substrate to dilute nutrient agar and observing for clearing around the colonies; iodine was added to the starch plate to detect the hydrolysis of starch. Cellulose hydrolysis was detected by the method of Peterson (11). Urease and deoxyribonuclease production were determined with Difco dehydrated media. Growth in Casitone- $\mathrm{Mg}^{2+}$ broth was determined by the method of McCurdy (9).

Optimum growth temperature. To find the growth temperature range and the temperature for optimum growth of $A$. disciformis CMU-1, dilute nutrient agar slants were inoculated and placed into an aluminum bar drilled with holes to accept the tubes and fitted with a cold plate on one end and a hot plate on the other. The apparatus covered the temperature range of 16 to $45^{\circ} \mathrm{C}$. After 3 weeks of incubation, the vegetative colonies were measured and the presence or absence of fruiting bodies was noted. Colony size and the presence of fruiting bodies were the criteria used to determine the optimum growth temperature.

Guanine plus cytosine $(G+C)$ content of deoxyribonucleic acid. Measurement by $\mathrm{CsCl}$ buoyant density of the $\mathrm{G}+\mathrm{C}$ content of the deoxyribonucleic acid extracted from $A$. disciformis CMU-1 was kindly performed by Manley Mandel, University of Texas System Cancer Center, Houston.

Electron microscopy and photomicrography. Specimens used for phase contrast and Nomarski in- terference photomicrography were prepared in wet mounts containing lactophenol. Specimens used for scanning electron microscopy (SEM) were fixed overnight in a fixative composed of ethanol, $356 \mathrm{ml} ; 40 \%$ formaldehyde, $72 \mathrm{ml}$; glacial acetic acid, $72 \mathrm{ml}$; glycerol, $36 \mathrm{ml}$; and distilled water, $500 \mathrm{ml}$. Fixed specimens were dehydrated overnight in acidified dimethoxy-propane followed by two changes of $100 \%$ acetone (10). Dehydrated specimens were then criticalpoint dried, sputter-coated with gold-palladium, and viewed in a Hitachi S-500 scanning electron microscope.

\section{RESULTS}

Isolated strain. To date, only one strain, designated CMU-1, of A. disciformis was obtained in pure culture. Although the organism was routinely enriched from a 1.5 liter sample of water and sediment obtained from Davis Lake in December 1977 and stored at $4^{\circ} \mathrm{C}$, further attempts to purify new strains of $A$. disciformis failed. Several strains originally thought to be axenic invariably contained a small, gram-negative contaminant.

Biochemical and physiological characteristics. $A$. disciformis CMU-1 was positive for Congo red dye absorption, starch hydrolysis in 3 days, casein hydrolysis, catalase production, and growth in Casitone- $\mathbf{M g}^{2+}$ broth. It was negative for cytochrome oxidase and urease production, deoxyribonucleic acid and cellulose hydrolysis, etching of agar, and growth at $40^{\circ} \mathrm{C}$. The growth range was 19 to $34^{\circ} \mathrm{C}$, with an optimum growth temperature of 28 to $30^{\circ} \mathrm{C}$. The $\mathrm{G}+\mathrm{C}$ content of the deoxyribonucleic acid (buoyant density in $\mathrm{CsCl}, 1.727 \mathrm{~g} \mathrm{~cm}^{-3}$ ) was $68.4 \mathrm{~mol} \%$.

Morphology. Vegetative rods of $A$. disciformis CMU-1 (Fig. 1A and 1C) were slender and flexible, slightly tapered at the ends, and measured 0.5 to 0.75 by 3 to 14 (average, 5 to 10 ) $\mu \mathrm{m}$. Whorls of gliding vegetative rods (Fig. 1A) were commonly observed on dilute nutrient agar. The vegetative colony grown on dilute nutrient agar was a thin, spreading swarm of gliding cells, pink to light orange in color. The colony often had concentric rings and radial folds emanating from the center. When the colony was scraped off the agar surface, the vegetative cells adhered together in the slime matrix which took on a distinct orange color. After 7 days of incubation at $30^{\circ} \mathrm{C}$, vegetative colonies grown on plates of dilute nutrient agar measured 1 to $2 \mathrm{~cm}$ in diameter. After 21 days, the colonies measured 3 to $5 \mathrm{~cm}$ in diameter.

Mature myxospores (Fig. 1B and 1D) were refractile and irregularly spheroid $(1 \mu \mathrm{m}$ in diameter) to oval (1.0 by $1.5 \mu \mathrm{m})$ in shape. Immature myxospores were observed as short rods (1 by 2 to $3 \mu \mathrm{m})$. The myxospores were contained within sporangia (Fig. 2A) which varied in shape 

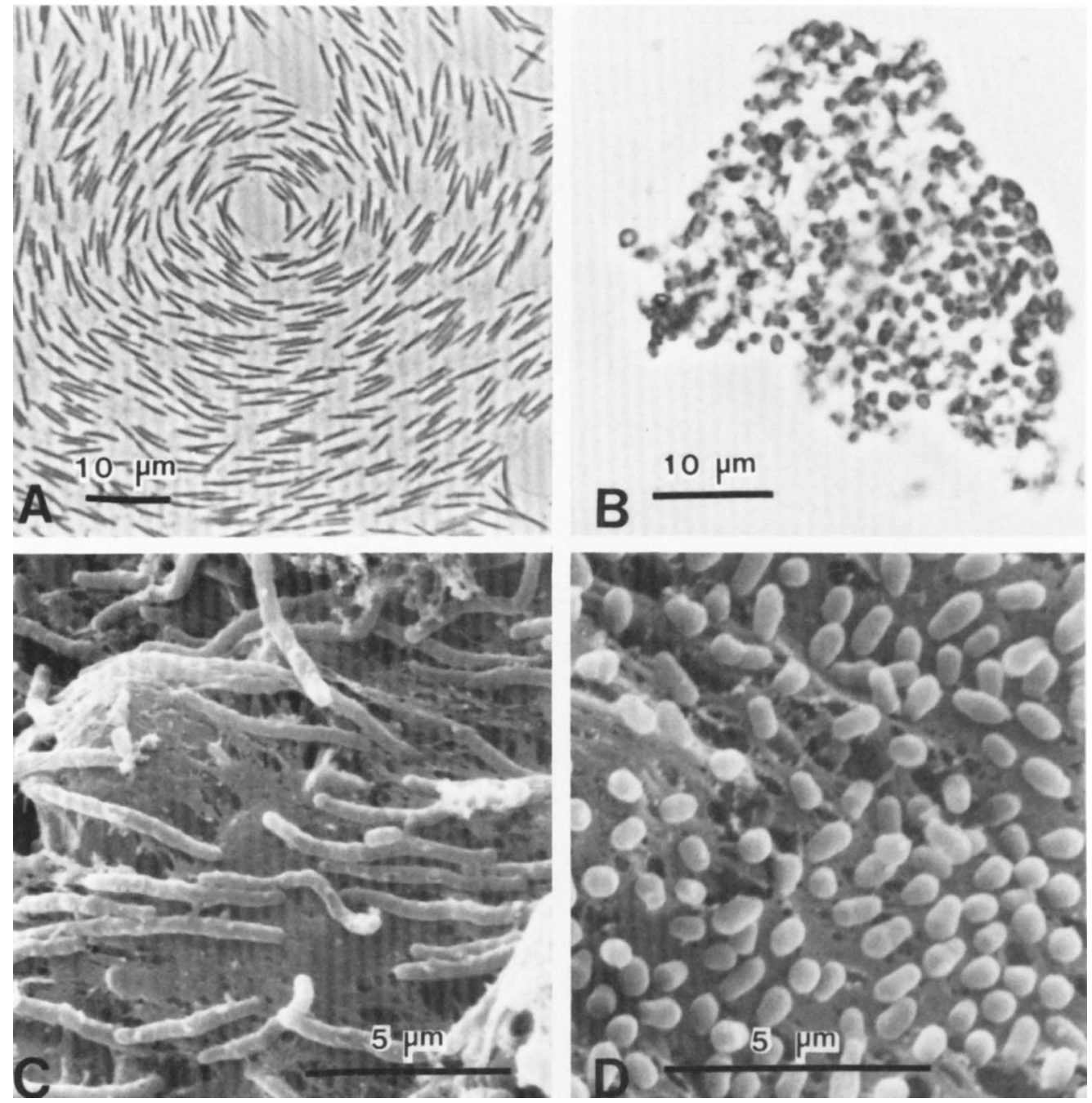

FIG. 1. Vegetative cells and myxospores of A. disciformis CMU-1. (A) Gliding whorl of vegetative cells (phase contrast, 1,250×). (B) Clump of myxospores released from a crushed sporangium (phase contrast, 1,250×). (C) Vegetative cells (SEM, 6,800×). (D) Myxospores (SEM, 8,300×).

and dimensions. Most sporangia were spheroidal in shape (Fig. 2B) and measured 25 to 30 by 30 to $45 \mu \mathrm{m}$ (average, 27 by $40 \mu \mathrm{m}$ ). A few sporangia were larger, measuring 30 to 40 by 50 to $75 \mu \mathrm{m}$. Many sporangia were flattened or disk shaped (Fig. 3C and 3D) and measured 10 to $20 \mu \mathrm{m}$ in thickness. Some sporangia were highly irregular in shape and were elongate, curved, or lobed (Fig. 2C and 2D). Occasionally, sporangia were borne on short sporangiophores (probably composed of slime) which measured 5 to 15 by 20 to $25 \mu \mathrm{m}$ (Fig. 2B and 5D). However, in general, the sporangia appeared to be sessile or attached directly to one another. The intact sporangium characteristically exhibited a scar of insertion
(Fig. 2B) at its point of attachment (13). After 6 to 8 weeks of growth on agar media, the sporangiophores often deliquesced, leaving the intact sporangium resting on the substrate.

A thin but rigid sporangium wall was present and appeared rough and convoluted (Fig. 3C) owing to its close adherance to the tightly packed myxospores within. The sporangium wall shown in Fig. 3C was partially peeled back, allowing the release of a few myxospores which remained attached to the sporangium. Sporangia could be crushed by applying thumb pressure on the cover slip of a wet mount, releasing myxospores which were often still attached to fragments of the sporangium wall (Fig. $3 \mathrm{~A}$ and 

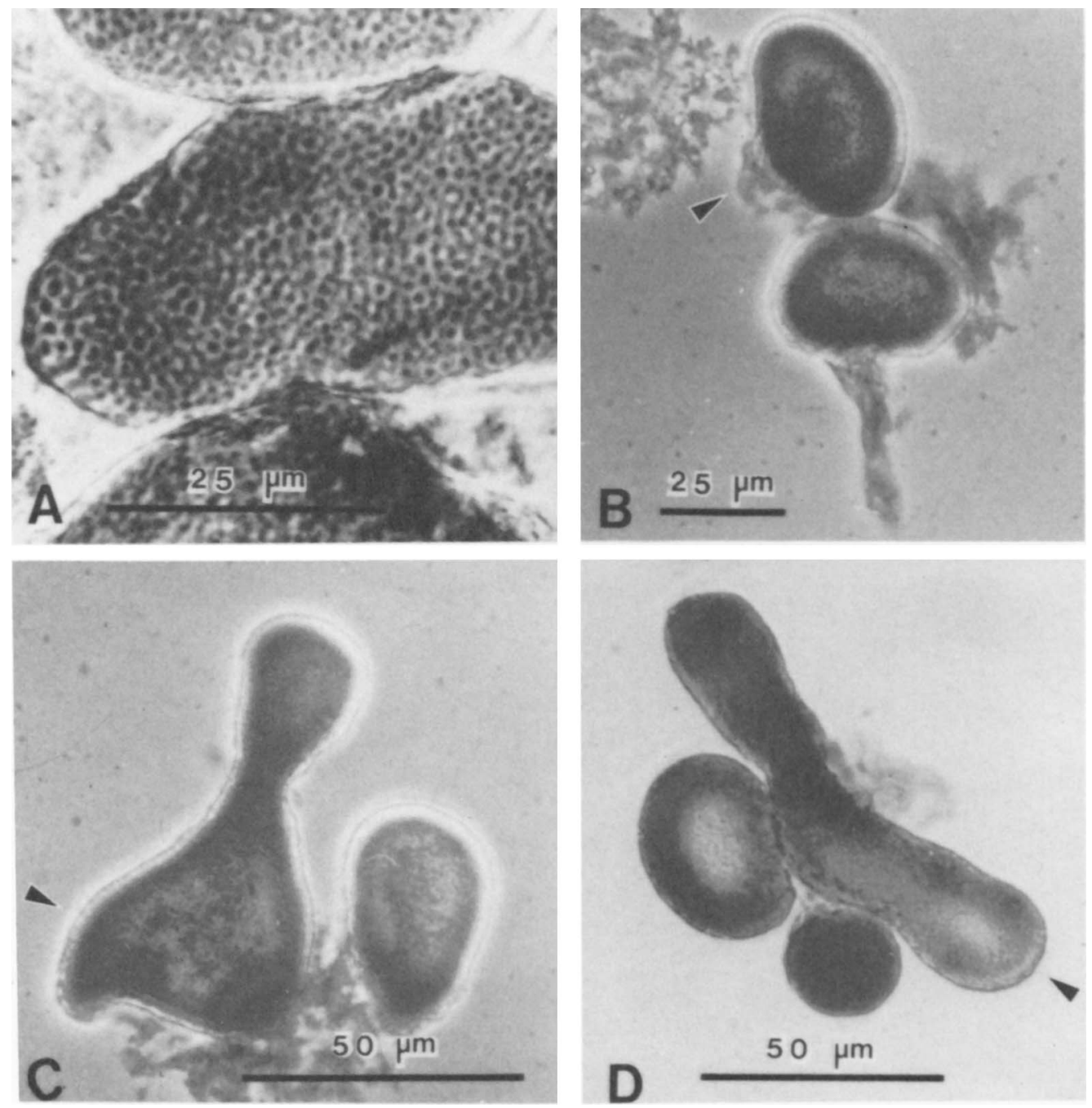

FIG. 2. Sporangia of A. disciformis CMU-1. (A) Sporangium stained with lactophenol-aniline blue. Note the spherical myxospores packed within the sporangium (brightfield, 1,250X). (B) Attachment of the sporangiophore. The arrow points to a scar of insertion. The lower sporangium is attached to a sporangiophore (phase contrast, 600×). (C) Irregular lobed sporangium (arrow) (phase contrast, 600×). (D) Irregular elongate sporangium (arrow) (phase contrast, 600X).

3B). Considerable thumb pressure was applied to this wet mount, indicating the strength of the sporangium.

The fruiting body of $A$. disciformis CMU-1 at maturity was characteristically orange in color and consisted of sporangia clustered in a heap (Fig. 3D and 5B). These features were seen invariably in the hundreds of enrichments examined. Immature fruiting bodies were pink to light orange in color. Each mature fruiting body contained from two or three to several hundred sporangia. Occasionally, solitary sporangia were observed attached directly to the substrate and set apart from larger fruiting bodies nearby. Fruiting body dimensions varied greatly, from the size of a single sporangium to a macroscopic mass several millimeters in diameter and up to $0.2 \mathrm{~mm}$ high.

Life cycle. Figure 4 shows the initial phases of $A$. disciformis CMU-1 fruiting body development on dilute nutrient agar. The vegetative rods first aggregated and oriented themselves in a parallel arrangement (Fig. 4A). As more cells reached the aggregation center, the mass of rods rose up off the substrate, forming a cushionshaped mass (Fig. 4B). Later, the mass began to 

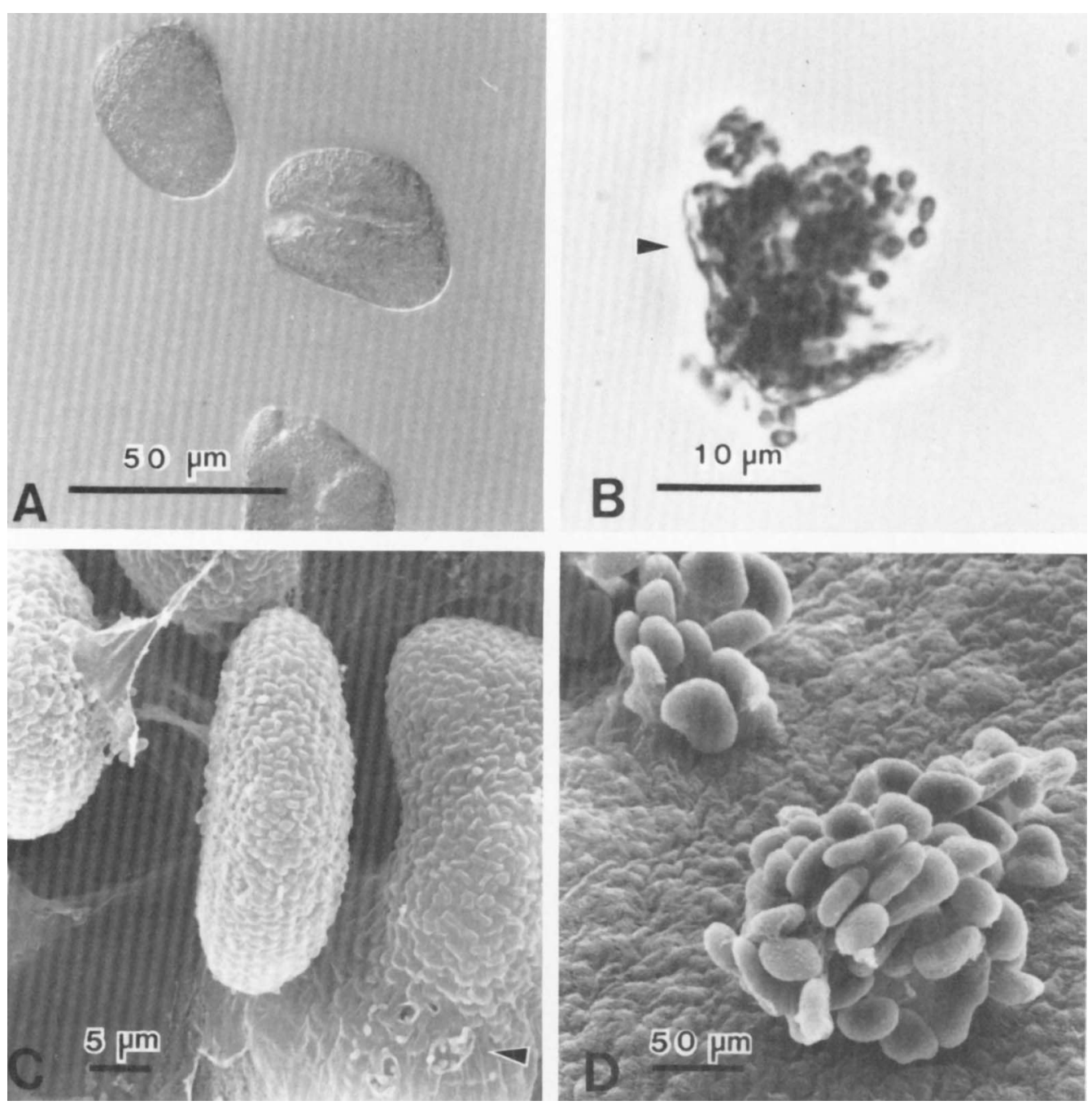

Frg. 3. Sporangia and sporangium wall of A. disciformis CMU-1. (A) Wet mount of crushed sporangia made by applying thumb pressure to the coverslip (Nomarski interference, $600 \times$ ). B. Fragment of sporangium wall (arrow) adhering to a clump of myxospores released from a crushed sporangium (phase contrast, 1,250 $\times$ ). (C) Disk-shaped sporangium viewed edge-on. The arrow points to a region where the sporangium wall was breached, releasing a few myxospores. Note the rough and convoluted texture of the surface of the sporangium (SEM, 2,000×). (D) Small, mature fruiting body containing several disk-shaped sporangia (SEM, 400X).

differentiate into two different regions: the emerging sporangia, which contained cells undergoing morphogenesis to myxospores, and the foundation below, still apparently composed of aggregating vegetative rods (Fig. $4 \mathrm{C}$ ). Clusters of sporangia appeared at the distal end of the fruiting body and were attached to the proximal foundation region (Fig. 4D). Figure 5A shows a fully differentiated portion of a developing fruiting body with four sporangia attached radially to a common central foundation composed of parallel-oriented vegetative rods. A higher-magnification detail of a foundation region (Fig. 5C) reveals slime fibrils attached to many cells. The mature fruiting body (Fig. 3D and 5B) consisted of a clump of sporangia heaped together in a clearly delineated mass. The positioning of the sporangia within the fruiting body did not appear to follow any discernible pattern.

\section{DISCUSSION}

A. disciformis is apparently a rare myxobacter. Peterson and McDonald (12) were unable to isolate the organism and doubted its validity as a species. The taxon was dropped from Bergey's 

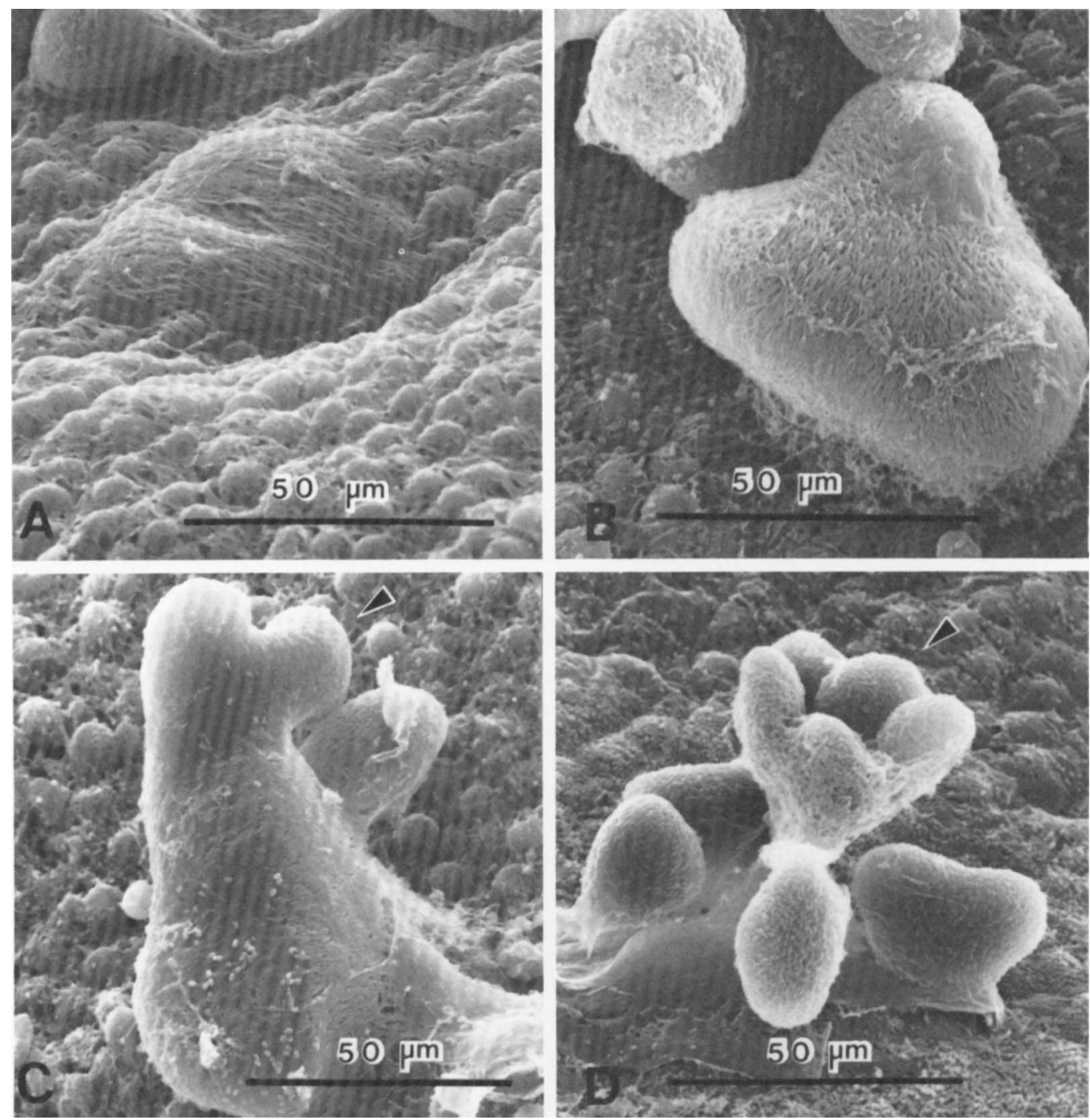

FIG. 4. Early phases of fruiting-body development in A. disciformis CMU-1. (A) Initial aggregation of vegetative cells at a fruiting center. Note the parallel orientation of the cells (SEM, 1,000X). (B) Accumulation of aggregating vegetative cells rising up off the substrate (SEM, 1,000X). (C) Initial differentiation of the fruiting body into emerging sporangia (arrow) at the distal end, and the foundation region at the proximal end $(S E M, 830 \times)$. (D) Cluster of maturing sporangia (arrow) attached to a foundation region (SEM, 900 $\times)$.

Manual in 1974 because no cultures were available and very little was known about the organism (9). The results of this and other studies $(3,6,7)$ indicate that the habitats of $A$. disciformis are restricted to specific ecosystems, which may explain why the organism has not been isolated until the present. $A$. disciformis was observed on numerous occasions in enrichments from Davis Lake, an ecosystem dominated by a quaking Spagnum mat (3). Sim:larly, the last previously reported observation of $\boldsymbol{A}$. disciformis was in forest soil and peat bog soil domi- nated by Sphagnum, Carex, and Vaccinium (7). All are plants commonly found in peat bogs. The original report by Thaxter (13) noted that the organism was found on muskrat dung, which suggests that water was nearby. It thus appears that $A$. disciformis may be associated with soils with a high moisture content, or with peat bog ecosystems in general. In Davis Lake, $A$. disciformis was routinely observed in all aquatic habitats of the bog, especially the fossa, but was not detected in the upland forest soil immediately surrounding the bog (3). Jahn (5) observed 

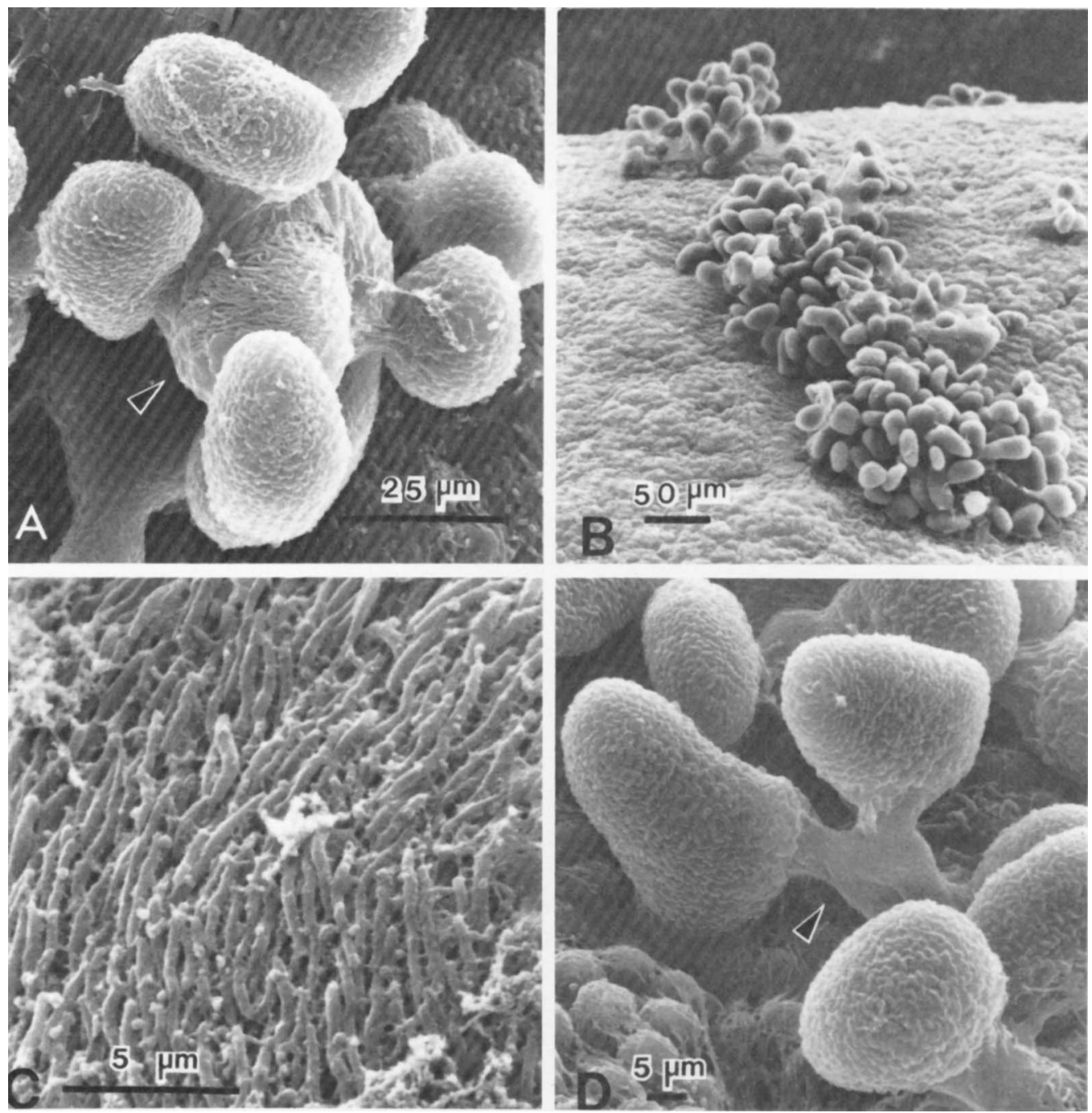

FIG. 5. Mature fruiting body of A. disciformis CMU-1. (A) Fully differentiated sporangia attached to a foundation region (arrow) (SEM, 1,000×). (B) Mature fruiting body (SEM, 200X). (C) Detail of foundation region showing parallel-oriented vegetative cells with attached slime fibrils (SEM, 5,600×). (D) Sporangia attached to a common sporangiophore (arrow) (SEM, 1,200×).

that $A$. disciformis was obtained only from acid soils within the $\mathrm{pH}$ limits of 3.6 to 6.4 . The lowest $\mathrm{pH}$ measured in Davis Lake was 6.0 and was probably the result of the addition of organic acids such as humic acid to the bog basin after the autumn leaf fall and rain run-off (3). Jahn (5) found that Polyangium sorediatum was only obtained from acid soils ( $\mathrm{pH} 3.6$ to 6.4). That organism was also observed frequently in the fossa region of Davis Lake but not in the surrounding soils (3). It will be necessary to obtain samples from a variety of peat bogs to determine whether or not $A$. disciformis and $P$. sorediatum (which has not yet been obtained in pure cul- ture) are part of the normal flora of these environments.

The morphology of $A$. disciformis CMU-1 described herein corresponds closely to that given in the original description by Thaxter (13) and to those by Jahn (4) and Krzemieniewska and Krzemieniewski (6). We believe that strain CMU-1 is identical to Thaxter's Myxococcus disciformis 1904 (13). The organism is clearly a myxobacter. It possesses a dimorphic life cycle which includes vegetative rods and myxospores, forms fruiting bodies, grows vegetatively in a slimy, swarming colony, is microbiolytic, glides on a solid substrate, and possesses a $\mathrm{G}+\mathrm{C}$ con- 
tent in its deoxyribonucleic acid of $68.4 \mathrm{~mol} \%$ (which falls within the range of 67 to $71 \mathrm{~mol} \%$ that is typical of the myxobacteria). According to the key to the families of the order Myxobacterales in the 8th edition of Bergey's Manual, A. disciformis belongs in the family Myxococcaceae by virtue of its spherical to oval myxospores (9). However, we are in agreement with Jahn (4) that the organism does not belong in the genus Myxococcus. The description of the genus Myxococcus, the only currently recognized genus in the family Myxococcaceae, specifies that "the myxospores are not enclosed in a sporangium" (9). Because $A$. disciformis possesses a distinct sporangium that contains the myxospores, the organism cannot belong to the genus Myxococcus under its present definition.

We therefore advocate that the genus Angiococcus Jahn 1924 be reestablished within the family Myxococcaceae and that the species $A n$ giococcus disciformis (Thaxter) Jahn 1924 be recognized as a valid species. We further propose that strain CMU-1, as described above, be accepted as the neotype strain of $A$. disciformis.

Family 1. Myxococcaceae Jahn 1924 (4). Genus 2. Angiococcus Jahn 1924 (4). An- gi. ococcus. Gr. noun angium, vessel; Gr. noun coćcus, a berry; M.L. mas. n. Angiococcus vessel coccus.

Vegetative cells are flexible, slender rods, slightly tapered at the ends. Motile by gliding. Refractile myxospores are irregularly spherical to oval in shape and are contained within distinct sporangia. Fruiting bodies consist of numerous sporangia in a pile that rises up off the substrate.

\section{ACKNOWLEDGMENTS}

We thank Manley Mandel, University of Texas System Cancer Center, Houston, for determing the $\mathrm{G}+\mathrm{C}$ content of the DNA of $A$. disciformis CMU-1.

This study was supported in part by the Central Michigan University School of Graduate Studies Research Grant and by the Department of Microbiology, Louisiana State Univer- sity. We acknowledge the use of the Louisiana State University Life Sciences electron microscopy facilities funded in part by Public Health Service grant SO7 RR007039-07 from the National Institutes of Health.

\section{REPRINT REQUESTS}

Address reprint requests to: Leonard A. Hook, Department of Microbiology, Louisiana State University, Baton Rouge, LA 70803 .

\section{LITERATURE CITED}

1. Brockman, E. R., and W. L. Boyd. 1963. Myxobacteria from soils of the Alaskan and Canadian Arctic. J. Bacteriol. 86:605-606.

2. Collins, C. H., and P. M. Lyne. 1970. Microbiological methods, 4th ed. Butterworth Publishers Inc., Boston.

3. Hook, L. A. 1977. Distribution of myxobacters in aquatic habitats of an alkaline bog. Appl. Environ. Microbiol. 34:333-335.

4. Jahn, E. 1924. Beitrage zur botanischen Protistologie. I. Die Polyangiden. Gebr. Borntraeger, Leipzig.

5. Jahn, E. 1936. Culture methods and metabolic studies with myxobacteria (Polyangium). Abderhalden's Handbuch d. biol. Arbeitsmeth. Abt. XII 1:921-925.

6. Krzemieniewska, H., and S. Krzemieniewski. 1926. Die Myxobakterien von Polen. Acta Soc. Bot. Pol. 4:154.

7. Krzemieniewska, H., and S. Krzemieniewski. 1927. Rozsiedlenie miksobacteryj. Acta Soc. Bot. Pol. 5:102137.

8. McCurdy, H. D. 1969. Studies on the taxonomy of the Myxobacterales. I. Record of Canadian isolates and survey of methods. Can. J. Microbiol. 15:1453-1461.

9. McCurdy, H. D. 1974. The gliding bacteria, p. 76-127, In R. E. Buchanan, and N. E. Gibbons (ed.), Bergey's manual of determinative bacteriology, 8 th ed. The Williams \& Wilkins Co., Baltimore.

10. Muller, L. L., and T. J. Jacks. 1975. Rapid chemical dehydration of samples for electron microscopic examination. J. Histochem. Cytochem. 23:107-110.

11. Peterson, J. E. 1969. Isolation, cultivation and maintenance of the myxobacteria, p. 185-210. In J. F. Norris and D. W. Ribbons (ed.), Methods in microbiology, vol. 3B. Academic Press Inc., New York.

12. Peterson, J. E., and J. C. McDonald. 1966. The demise of the myxobacterial genus Angiococcus. Mycologia 58: 962-965.

13. Thaxter, R. 1904. Contributions from the cryptogamic laboratory of Harvard University. LVI. Notes on the Myxobacteriaceae. Bot. Gaz. 37:405-416. 